\title{
L'argent n'apporte pas le bonheur mais il achète la liberté : carrière et refus du bonheur conjugal chez les personnages femmes de Malika Mokeddem
}

\section{Simona Emilia PRUTEANU, Université Wilfrid Laurier}

Depuis la parution de son premier roman, Les hommes qui marchent (1990), Malika Mokeddem, écrivaine d'origine algérienne établie en France, crée des personnages de femmes migrantes qui s'opposent aux enfermements, que ce soit dans un territoire ou dans les carcans de la tradition. Alors que la France représente l'espace de l'écriture, l'image de l'Algérie ${ }^{1}$ revient constamment dans les romans de Mokeddem, à travers l'évocation de ses origines dans le désert et surtout par la transgression des plus élémentaires rites de passage associés à ces origines. Si toutes les filles algériennes doivent faire l'apprentissage de l'obéissance, du mariage et de l'enfantement, les héroïnes de Mokeddem incarnent le refus de ces lois de la tribu : douées pour les études, elles en font leur planche de salut et parviennent ainsi à repousser indéfiniment la date fatidique d'un mariage arrangé. Plus tard, le salaire qu'elles gagnent leur assure l'indépendance financière et le droit de dire non aux exigences de la famille qu'elles font vivre, même de loin.

Le but de cet article est donc de s'interroger sur les rapports d'oppositions qui marquent l'existence des personnages féminins de Mokeddem, oppositions qui viennent autant de l'intérieur du personnage que de son extérieur. C'est pourquoi on trouvera souvent que le même élément peut faire figure d'objet positif et négatif selon les circonstances. Par exemple, le tout premier obstacle au bonheur personnel des narratrices dans les romans de Mokeddem est représenté par le manque d'affection et de soutien de la part de leurs familles; néanmoins, l'amour véritable personnifié par l'homme aimé sera à son tour perçu par l'hérö̈ne comme un autre type d'assujettissement, une attache qu'elle coupera la première ${ }^{2}$. Dans ce cas, manque d'affection ou trop plein de l'amour s'opposent en égale mesure à la quête de liberté de la femme. L'argent est un autre élément qui joue un double rôle dans la vie des narratrices : tout en refusant de se laisser acheter par un potentiel mari ou amant, la femme travaille dur pour atteindre une stabilité économique. L'argent qu'elle gagne et qu'elle verse à ses parents lui donne le droit de leur dire «non », puisqu'elle assume ainsi un rôle traditionnellement masculin, et, dans ce cas, l'argent est vu comme symbole de liberté. Pourtant, lorsque l'argent, sous toutes ses formes - prêt, don, cadeau - leur est offert par quelqu'un d'autre, même s'il s'agit des 
personnes aimées par les narratrices de Mokeddem, elles s'en méfient et le refusent, puisqu'elles s'attendent à ce qu'on leur exige de renoncer à quelque chose en échange, notamment à une partie de leur liberté difficilement acquise.

En outre, sur les dix romans publiés par Mokeddem jusqu'à date, aucune héroïne ne connait la sérénité dans le couple pendant longtemps et elles rejettent toutes la maternité. Nous nous interrogerons donc sur les raisons de ces absences aussi bien que sur leur rapport à l'argent, afin de voir si on peut y lire une affirmation absolue de la liberté de choix de la femme plutôt qu'un échec causé par les circonstances de l'immigration. Tous ces rapports et questionnements sont repris par Mokeddem dans son plus récent roman, La désirante (2011), où, pour la première fois dans l'évolution romanesque des personnages de Mokeddem, l'héroïne se laisse protéger, aimer, et semble avoir atteint la maturité et la stabilité émotionnelles. Tel que nous le montrerons plus loin, tout cela passera par une auto-analyse qui aura pour but de démêler le rapport complexe que Shamsa entretient à l'égard de l'argent lorsque celui-ci s'insinue dans ses relations intimes.

\section{Famille et argent}

Les relations familiales, tout aussi bien que leur absence, mettent leur empreinte sur le développement de tous les personnages de Mokeddem; qu'elles soient étouffées par la surprésence des pères ou des frères autoritaires, ou hantées par l'absence de la mère, morte ou simplement distante envers sa fille, les héroïnes se construisent surtout contre l'image de la femme que la famille attend qu'elles deviennent ${ }^{3}$. Séparées volontairement de leurs familles suite à leur immigration, et choisissant de ne pas enfanter, ces femmes sont pourtant le point de départ d'un nouveau type de filiation spirituelle. Ce nouveau type de filiation réunit à travers l'écriture l'héritage féminin d'une génération qui n'a pas eu le droit à la parole, à savoir les grand-mères et les mères des héroïnes, et l'héritage culturel mixte que les narratrices migrantes sont en train de se construire sur l'autre rive de la Méditerranée. Une fois libres de s'exprimer, c'est en tant que femmes que les personnages de Mokeddem prennent la plume et créent ce que Julie Brunet appelle de véritables romans familiaux au féminin :

Nous ne sommes pas ici en présence du modèle classique freudien où un sujet «neutre » tente de se libérer de l'origine et des liens familiaux en les réinventant, mais d'un modèle féminin où un sujet, en l'occurrence une femme, cherche à échapper aux origines familiale et culturelles patriarcales, aux traditions 
entourant la féminité et la maternité, aux rapports de sexe et de pouvoir en vigueur dans le pays natal. (97)

La famille est, cependant, inévitablement liée dans les écrits de Mokeddem à l'idée des relations corrompues par le poids de la tradition et de l'argent, et aucune des figures parentales n'est décrite avec plus de sympathie que l'autre. D'une part, la communauté féminine reçoit une représentation ambivalente dont le référent est l'attitude que les femmes sont obligées d'adopter dans la société d'après leur âge. Dépourvues de pouvoir, et même du droit de choisir leur propre sort, les jeunes femmes doivent apprendre à se soumettre à la volonté de leurs parents d'abord, plus tard à celle de leurs frères et ensuite aux décisions de la famille de leur époux. C'est lorsqu'elles deviennent âgées qu'on leur accorde le respect et que toute la famille se réunit autour de la plus vieille considérée comme étant la plus sage. Selon Mokeddem, les mères ne font que perpétuer une tradition oppressive envers leurs filles au lieu de les protéger parce qu'elles associent ce parcours à un « apprentissage au bout duquel elles obtiennent le droit de régner sur une tribu » (Helm 46).

Les hommes qui marchent décrit cette hiérarchie féminine au sein de la famille et la relation qui s'établit entre les trois générations, la grand-mère Zohra, sa bru Yamina et la fille aînée de celle-ci, Leïla ${ }^{4}$. Zohra, ancienne nomade, vit à travers les souvenirs des longues marches sillonnant le désert, histoires que sa petite-fille ne se fatigue pas d'écouter. De cette manière, la grand-mère se situe toujours entre deux temps et deux espaces, à savoir le passé nomade vécu dans les dunes et son présent sédentaire dans la maison de son fils. Apparemment immobilisée dans l'espace, Zohra maîtrise l'art de conter ou ce qu'elle appelle au début du roman «le nomadisme des mots » (11) et elle insuffle à sa petite-fille le désir de se déplacer. Le royaume de l'oralité où règne sa grand-mère, fournit à Leïla un premier espace-refuge car, non seulement elle s'échappe dans le monde des contes de Zohra, mais elle emprunte le vocabulaire coloré de celleci pour tenir tête à sa mère: «Je ne veux plus être ton ouvrière, ton esclave, reine de ruche ! hurla-t-elle au visage de sa mère, usant des métaphores savoureuses de son aïeule » (121).

Si Les hommes qui marchent ne présente pas une mère «idéale » dans les traits de Yamina, la communauté féminine se rachète par quelques figures très présentes dans la vie de Leïla : madame Bernard, l'accoucheuse française, marginalisée par ses concitoyens pour les amitiés qu'elle entretient avec les Algériennes, l'institutrice Madame Bensoussan qui encourage Leïla d'aller au lycée, et surtout Emma Beb Yatto. Emma est la mère de Sarah, l'amie juive de 
Leïla, et c'est dans la maison et dans les bras de celle-ci que Leïla reçoit l'affection maternelle qui lui fait tellement défaut : «Leïla se jetait contre sa poitrine et enfouissait la tête au plus chaud de sa gorge opulente. [...] Le giron de sa propre mère, toujours rempli par le gros ventre ou occupé par un dernier-né, toujours assailli, entouré par d'autres bambins, lui restait inaccessible » (157). Quant à la figure du père dans le roman, celui-ci ne semble valider l'existence de sa fille qu'au moment où celle-ci lui apporte un gain financier, au lieu de lui coûter la dot habituelle. Mais l'image paternelle est surtout liée au mensonge et à la déception : le père demande à Leïla de s'occuper de son petit-frère qui a besoin de soins spéciaux et, en vrai esprit ouvert, accepte de la payer pour ce travail afin qu'elle ne se sente plus exploitée. L'argent que Leïla amasse difficilement est destiné à l'achat d'une bicyclette qui rendrait son trajet à l'école moins pénible et que le père refuse de lui acheter sous prétexte qu'il n'en a pas l'argent. La rage que Leïla éprouve lorsqu'elle découvre sa tirelire cassée, n'est pas seulement provoquée par le vol de l'argent mais aussi par la certitude qu'on lui a fait cela en vertu de sa position inférieure dans la famille en tant que fille : « Tu n'es plus mon père, je t'ai fait confiance, et tu m'as trahie. Je te hais ! Je te hais ! tu n'aurais jamais fait ça à l'un de tes fils. Je le sais, et je te hais encore plus pour ça!» (143)

Lorsque la fille devient indépendante et même subventionne sa famille avec ses gains, elle s'attire le consentement du père qui l'élève au même rang que lui et ses fils, tel qu'on peut lire dans le roman biofictionnel La transe des insoumis (2003) : « Ma fille, maintenant tu es un homme...» (136). Cette réplique du père, censée flatter de manière hypocrite la fille qui lui remet tout son argent, exprime en fait le rapport d'esclavage dans lequel sont maintenues les femmes : dépourvues d'éducation et d'argent, elles doivent obéir aux hommes et payer pour leur existence de leur travail et soumission à la maison. Par contre, grâce à son autonomie financière, Malika a la chance de devenir une « esclave affranchie » : « salaire après salaire, je consolide des acquis. J'achète ma liberté » (136).

Néanmoins, avec l'émancipation, un fossé de plus en plus profond se crée entre les personnages de Mokeddem et leurs familles. Le personnage principal du roman Je dois tout à ton oubli (2008), le docteur Selma Moufid, se perçoit comme une étrangère parmi sa grande tribu et n'a pas le sentiment d'avoir eu la même mère que sa nombreuse fratrie : «Du reste, elle n'a pas plus fréquenté ses autres frères et sœurs. À la fin de la journée, ils rentraient tous chez leur ${ }^{5}$ mère... Selma, elle, s'enfermait à l'internat même les samedis, dimanches et autres jours fériés » 
(53). Après la mort de son père, Selma fait vivre la famille avec le peu qu'elle gagne en tant qu'institutrice à l'internat, « remplaçant le père aux cordons de la bourse familiale » (121). Ce sacrifice ne lui semble pas trop grand, vu qu'il lui permet de couper encore plus ses attaches, jusqu'au point où l'envoi de l'argent représente l'unique contact avec la famille : « Même loin, Selma n'a jamais dérogé à ce devoir. Cela la dispensait de l'obligation de revenir mais rendait encore plus effroyable son constat : s'acquitter de ce tribut était la seule expression du lien familial » (58). De son côté, Jane E. Evans voit dans ce rapport financier une stratégie de passage sous silence, qui arrange autant Selma que son clan. Alors que la famille ne veut rien savoir de sa vie en France, et surtout de sa relation avec un Français, Selma non plus n'accueille leur ingérence dans ses affaires, c'est pourquoi sa famille n'apprendra jamais qu'elle est en fait séparée depuis longtemps : «[...] Selma exchanges monthly checks for her family’s disinterest in her life » (189).

Les relations familiales que les autres perçoivent comme un gage de sécurité affective et financière sont ressenties par les héroïnes de Mokeddem soit comme des rapprochements forcés, ayant comme seul but d'étouffer leur liberté individuelle, soit comme l'expression d'une cohésion trompeuse, derrière laquelle la famille cache ses ignominies. Après la perte d'une patiente, Selma commence à se remémorer un épisode traumatique de son enfance qu'elle a refoulé pendant un demi-siècle : l'infanticide du nouveau-né de sa tante Zahia, crime perpétré par la mère de Selma. Étant donné que la relation avec sa mère a toujours été tendue, Selma n'est pas sûre qu'il s'agisse d'un vrai souvenir ou d'un cauchemar. C'est pourquoi elle décide de rentrer et de confronter sa mère, tout en jetant un regard extérieur sur le reste du clan dont elle se délimite nettement : «Elle regarde les membres de cette famille à l'apparence unie. Certains d'entre eux connaissent-ils le lourd secret ? Partagent-ils d'autres indignités à l'insu de Selma ? » (54). Lorsque Selma se retrouve seule avec sa mère, elle accuse celle-ci d'avoir étouffé le nouveau-né de sa tante Zahia, tout en espérant un démenti catégorique. Cependant, la mère avoue d'un seul trait son crime et son impuissance : «Qu'est-ce que tu voulais qu'on fasse ? On était bien obligés de tout étouffer !»(61). En essayant d'expliquer la lâcheté de sa famille, Selma a du mal à voir dans l'acte de l'infanticide une réparation de l'honneur du clan ; pour elle, il ne s'agirait que de «l'autocensure, l'autopunition, l'inflexibilité d'une tradition obscurantiste » (66). Le retour de Selma dans le désert et la confrontation avec sa mère donnent lieu à une réflexion sur la condition de la femme algérienne, réflexion qui se poursuit sur deux plans 
temporels : d'une part on a accès aux souvenirs de Selma, de l'autre, aux constatations qu'elle fait lors de son séjour. Malheureusement, les deux visions coïncident toujours dans la description du sort réservé aux femmes, sauf que le regard que Selma pose maintenant sur celles-ci est plus compréhensif. Si sa mère et ses tantes s'avèrent coupables de l'infanticide, elles ne sont pas les seules femmes poussées à de tels actes par la lâcheté des hommes :

Une sinistre certitude vient plomber davantage encore la nuit de Selma : avec une population qui a plus que triplé depuis l'indépendance, l'exode rural massif, la paupérisation, le manque de logements qui fait s'entasser plusieurs générations d'une même famille dans des espaces exigus, l'Algérie doit battre tous les records en nombre d'incestes. Et d'infanticides. Mais cela ne relèvera jamais d'aucune statistique. (66)

Alors que Mokeddem dénonce le passage sous silence de ces abus contre les femmes et les enfants, dans son étude sociologique intitulée The Eloquence of Silence : Algerian Women in Question (1994), Marnia Lazreg estime que les femmes algériennes ont toujours agi à travers leur histoire de façon à rendre leur silence bien éloquent. Le silence de la mère de Selma relèverait selon la catégorisation de Lazreg - silence circonstanciel, silence structurel et silence stratégique - du domaine du silence stratégique, qui représente un acte volontaire d'autodéfense : «Strategic silence was and still is a voluntary act of self-preservation when a woman feels it is better to keep quiet than to incur someone's wrath or disapproval » (18).

Bien qu'elle ne cherche pas à justifier le meurtre, Selma doit se rendre à l'évidence que les femmes comme sa mère et sa tante Zahia n'auraient pas eu de chance de survie sans avoir gardé le silence, tout en subissant des pénitences pires que l'emprisonnement, devant vivre avec le poids de ce crime, « la pire manière d'annihiler des mères, de tuer une part d'elles-mêmes » (72). La confirmation du secret a pour Selma un effet libérateur sur plusieurs plans : d'une part, elle explique mieux cette « relation âpre, sans la moindre tendresse » (120) entre elle et sa mère ; d'autre part, si l'existence de ce secret a scindé la personnalité de Selma, c'est aussi ce qui a alimenté sa rébellion et son esprit indépendant : «On prétendra que Zahia a fait une faussecouche. Ce mensonge va introduire une dissonance dans mon esprit sans que je n'en sache jamais la provenance. C'est le seul motif avoué de ma défiance... » (33). En outre, être capable de mettre un tel traumatisme derrière elle, même à cinquante-cinq ans, permettra à Selma, selon Evans, de réconcilier les différentes parties de son identité : «One can interpret this act as her first step towards psychological reintegration and an enhanced ability to love and be loved» 
(105). La fin du roman et la joie de vivre renouvelée de Selma préfigurent en quelque sorte le personnage de Shamsa, protagoniste du roman La désirante.

À la lumières de ces traumatismes, il est plus facile de comprendre pourquoi les narratrices de Mokeddem, sans refuser la vie en couple, refusent l'idée de mariage et la maternité ${ }^{6}$, comme pour essayer de se soustraire à l'héritage taché de leur filiation féminine. Dans sa biofiction, La transe des insoumis, Mokeddem semble suggérer que si Malika s'est mariée avec Jean-Louis, son époux pendant dix-sept ans, ce n'était que pour des raisons administratives qui concernaient son permis de séjour en France : « [...] je déteste le mot mari. Je ne l'emploie jamais. Je préfère dire compagnon » (199). La plupart de ses narratrices vivent en France avec un compagnon français et chrétien, ce qui signale une autre rébellion de leur part contre la tradition : le Coran stipule que, si un homme peut prendre pour épouse une femme qui n'est pas musulmane, les femmes n'auront jamais le droit d'épouser un « mécréant » ${ }^{7}$.

\section{Mère et maternité}

Quant à la maternité, l'écrivaine a avoué dans son essai autobiographique Mes hommes (2005) avoir eu recours à l'avortement, un choix douloureux mais qu'elle ne regrette pas, étant donné son désir plus grand d'indépendance : «Mon avortement, il y a combien de temps ? Combien d'années ? Je compte. Je me trompe. Je recompte. Ce n'est ni un sentiment de culpabilité ni de remords. C'est une cicatrice de ma liberté » (184-185).

Les raisons du refus de la maternité se justifient en grande partie par la mauvaise relation que les narratrices de Mokeddem ont avec leur mère, relations qui miroitent celle que Mokeddem a eue avec sa propre mère. Leïla, personnage principal des Hommes qui marchent, est, à l'instar de Mokeddem, l'aînée d'une nombreuse fratrie et se sent orpheline de mère parce que celle-ci n'a jamais de temps pour l'affection, mais seulement pour les réprimandes. Exaspérée par ses multiples grossesses, Leïla appelle sa mère Yamina «une usine à enfants » (116), ce qui n’est pas loin de la réalité, puisque la nombreuse progéniture sert à protéger leur mère d'une possible répudiation et lui accorde du pouvoir dans la famille : «Yamina s'était mise à exister grâce à son ventre. C'est grâce à lui qu'elle avait parfois droit au chapitre de la protestation » (116). Ce manque de mère est éprouvé par Selma aussi, qui, de ce fait, sent qu'elle ne peut pas s'inscrire dans une filiation féminine : «Elle n'a jamais eu de mère et elle ne sera jamais mère ${ }^{8}$ » $(138)$. 
C'est donc aussi dans le désir de se construire contre l'image de la mère que les narratrices rejettent la maternité, un choix qui, s'il ne fait pas souffrir la mère, du moins la contrarie et la met mal à l'aise dans son entourage féminin, pour lequel la question «combien astu d'enfants ?»(La transe, 181) tient lieu d'introduction. Dans La transe des insoumis, le personnage de Malika explique ainsi la psychologie de sa mère : «Qu'une femme puisse refuser d'enfanter est de l'ordre de l'inimaginable, de l'inconcevable pour [sa mère]. [...] n'avoir pas d'enfant représente la plus terrible catastrophe quelle qu'en soit la cause » (181).

Alors que le refus de la maternité reste une constante de la création littéraire de Mokeddem, dans son plus récent roman, La désirante, elle renouvelle son traitement de la thématique du couple et de son rapport à l'argent en construisant un personnage qui se laisse peu à peu apprivoiser par le bonheur et qui parvient à surmonter ses préjugés. Née dans le désert, Shamsa ${ }^{9}$ a été abandonnée dès la naissance par ses géniteurs et elle a été recueillie par des bonnes sœurs, dont Bernadette deviendra sa mère de substitution. Devenue journaliste, Shamsa décide de se réfugier en France au moment où la guerre civile en Algérie bat son plein : «Je n'en pouvais plus de voir l'Algérie se détruire, semer la panique et la misère. [...] Je partais pour ne pas disparaître à mon tour comme disparaissait mon passé » (100-101). Elle laisse derrière elle son amant depuis quatre ans, Zin, lui aussi journaliste, marié et père de trois enfants. Des années plus tard, Shamsa explique ce choix de relation illicite à son compagnon français Léo, qu'elle surnomme Lou : «Avant toi, l'amour ne me semblait qu'une mièvrerie propre à faire avaler toutes les couleurs du conformisme. Le sexe, oui. Pas les chaînes des conventions » (88). Au moment où la narration commence, Léo est disparu en Méditerranée depuis huit mois et ce n'est que vers la fin du roman qu'on apprend qu'il avait été enlevé par des trafiquants d'armes. Au début de leur enquête, les soupçons des policiers tournent autour de sa compagne, qui est choquée d'apprendre qu'elle est l'unique bénéficiaire de l'assurance-vie de Léo. C'est le père de Léo, Régis, qui, étant au courant de la situation, défend Shamsa de cette accusation en expliquant aux policiers que son fils ne voulait pas embarrasser sa compagne, qui aurait pu disposer de toute sa fortune si seulement elle l'avait désiré : «Léo tenait tellement à ce qu'ils se marient. Il aurait volontiers fait rédiger un acte notarié de donation au dernier vivant. Du reste, il n'a pas renoncé au projet d'en convaincre Shamsa. Et j'espère qu'il y réussira » (46).

Le fait que Léo soit issu d'une famille fortunée, avait poussé Shamsa au début de leur relation à battre en retraite, puisque sa richesse lui rappelait encore plus son altérité à elle : «le 
faste de ta maison me renvoyait d'emblée au statut ingrat de l'intruse, doublement étrangère » (80). Elle se rend compte qu'elle a toujours fui les gens qui avaient de l'argent comme si leur proximité représentait une attaque à sa liberté, sans trop savoir les causes de ces préconçus. En analysant sa relation avec Léo, elle conclut que c'est probablement à cause des rapports malsains dont elle avait été témoin en Algérie : «Et je tournais le dos. Sans doute parce que je ne concevais ces relations-là que cousues au fil empoisonné du fric, des traditions et des conventions » (79). Bien que leur relation évolue, Shamsa s'oppose toujours à une conception du mariage en tant que forme de «protection » de la femme et refuse les demandes de Léo : « Tu avais si peur que je reste 'démunie' s'il t'arrivait 'quelque chose' que tu t'échinais à me convaincre que nous devions nous marier » (103).

La relation de Shamsa avec les parents de Léo évolue elle aussi après la disparition de celui-ci. Craignant leurs soupçons quant à ses intentions, elle avait refusé de les rencontrer pendant les premiers deux ans de sa relation avec leur fils, et leur premier déjeuner à quatre s'était avéré un échec. Si le père de Léo se montre très accueillant et chaleureux, la mère, Caroline, profite des apartés avec Shamsa pour lui «lancer des piques venimeuses à souhait : je ne serai sûrement pas la 'dernière amie' de Léo » (133). La crispation des rapports entre les deux femmes n'est pas seulement le résultat du comportement de Caroline mais aussi des barrières dont Shamsa s'entoure, puisqu'avoir une famille, des parents, est un territoire inconnu et dangereux pour elle : «Comme si la vie trouvait enfin le moyen de se jouer de mes défenses, me forçant ainsi à une proximité avec ce qui m'est le plus étranger chez une femme : la maman » (129).

Après l'épisode du déjeuner chez les parents de Léo, Shamsa refuse de remettre pied chez eux pendant deux autres années, pendant lesquels Caroline essaie de se faire pardonner en lui montrant un autre visage et en la comblant de présents : «Elle arrivait avec une montagne de cadeaux pour son fils, pour moi surtout. Elle s'empressait d'approuver chacun de mes propos, me témoignait une attention de tout instant » (133).

La réticence de Shamsa d'accepter de tout cœur les efforts de Caroline n'est pas sans rappeler «L'essai sur le don » (1968) de Marcel Mauss qui affirme que le don, en tant que fait social, présuppose une acceptation de la part du destinataire, puisque accepter c'est s'obliger à rendre la pareille. En outre, selon Mauss, la chose donnée, même si elle a été volontairement abandonnée par le donateur, continue à l'incarner en quelque sorte et à prolonger son influence 
sur le récipiendaire. Si Shamsa craint les élans généreux de Léo et de sa famille, c'est parce qu'elle craint leur emprise sur son indépendance ${ }^{10}$. Après la disparition de Léo, il y a un rapprochement entre Shamsa et Caroline qui se réconfortent mutuellement, jusqu'au point où Caroline commence à se comporter en mère despotique envers Shamsa, qui se sent suffoquée par cette intrusion dans sa vie : «[...] je ne supporte plus ses incessantes pleurnicheries, son ingérence, son chantage - sa peur que je parte -, ses débordements, ses visites impromptues » (129). Vexée par ce qu'elle perçoit comme un manque d'empathie de la part de Shamsa, Caroline essaie de la blesser en soulignant un fait trop évident, notamment l'absence de la maternité dans la vie de Shamsa, due en partie à sa condition d'orpheline et en partie au refus d'enfanter : «Tu réagis comme ça parce que tu ne sais pas ce que c'est une maman » (130). Les paroles de Caroline font écho à la pensée de Daniel Sibony qui, dans son ouvrage Entre-deux. L'origine en partage, attire l'attention sur l'importance de la transmission de l'héritage féminin, et les conséquences de ce manque : «Une femme à qui sa mère n'a 'rien donné' ne pourrait être ni fille ni mère et resterait en suspens de son être-femme, dans son entre-deux langue à elle, ne pouvant ni 'lire', ni 'écrire' les traces de sa féminité » (63).

Qualifiée par Shamsa de «mère abusive », Caroline ne tarde pas à lui demander pardon. Les pensées de Shamsa trahissent le malaise du personnage envers l'existence possible de l'amour inconditionnel et du pardon; n'ayant pas connu ce genre de rapport familial, Shamsa préfère ne pas y croire et maintient une attitude sceptique, et possiblement erronée, sur les motivations du geste de Caroline: «Ce genre de réaction me donne le sentiment que l'indulgence et la bonté, ne sont, parfois, que des formes édulcorées de l'arrogance » (130).

Néanmoins, pendant l'année où Léo est absent, sans qu'on sache s'il est mort ou encore vivant, Shamsa, Caroline et Régis se retrouveront en véritable famille liée par l'amour et par l'espoir de retrouver Léo. Lorsque Shamsa entreprend de partir en voilier à la recherche de pistes sur la Méditerranée, elle accepte la bénédiction de Caroline, matérialisée dans un bien de famille qui scelle son inscription dans l'arbre généalogique de la famille de son amoureux : «Shamsa, ma fille, j'aimerais que tu mettes cette bague. Elle appartenait à ma grand-mère. Elle te protégera » (12). C'est d'un autre œil que Shamsa regarde maintenant la générosité des parents de Léo envers elle, puisqu'elle a appris à les aimer à son tour, à apprécier l'importance de ce lien affectif dans sa vie, et la vulnérabilité à laquelle ces attaches l'exposent. Avant de quitter l'Algérie, sa condition d'orpheline célibataire était une armure qui la protégeait contre le 
désespoir qui guettait tous ceux qui avaient quelqu'un ou quelque chose à perdre : «moi, je n'ai pas de mari. Pas de frère. Pas de fils. Pas de fille. Personne à enlever, à torturer, à tuer » (18). Maintenant, en l'absence de Léo, elle se sent réconfortée par l'amour de ses deux parents à lui, qui sont devenus, en quelque sorte, ses parents à elle : «Ce soir, j’ai tellement envie de me jeter dans leurs bras, ils me manquent tant qu'avant de raccrocher, je m'entends leur chuchoter, presque à mon insu : 'Je vous aime'. Ils en restent sans voix » (170).

\section{Conclusion}

Plus de vingt ans séparent la publication du roman Les hommes qui marchent de celle de La désirante. À cet effet, le but de cet article a été de montrer le changement, qui, à notre avis, s'est opéré peu à peu dans l'évolution psychologique des personnages de Mokeddem : si ses premières narratrices sont des jeunes filles se rebellant contre l'autorité familiale et contre le mariage associé à la prison, ses héroïnes plus récentes ont subi une maturation physique et intellectuelle. Elles sont toutes des femmes indépendantes, dans la trentaine, la quarantaine ou même la cinquantaine, tel le personnage de Selma. Leurs conceptions de la vie en couple, de la dépendance affective et financière semblent avoir dépassé le rapport de pouvoir inégalitaire homme/argent - femme/soumission qui caractérisait les premiers titres de Mokeddem. On peut voir une autre ligne de démarcation, géographique notamment, entre ces deux types de personnages : les plus jeunes s'apprêtent à quitter l'Algérie pour la France ou viennent tout juste d'arriver en France, mais leur destin reste ouvert à l'imagination des lecteurs : «one can only speculate whether Dalila of L'interdite, Dounia of La nuit de la lézarde, and Selma's intellectually gifted niece overcome, through determination, struggle, and education, Algerian society's stifling of young women's creative intelligence » (Evans 109). Par contre, Mokeddem offre à ses narratrices adultes et habitant la France depuis des décennies, des dénouements dignes de personnages plus complexes ayant expérimenté la vie des deux côtés de la Méditerranée.

Sa toute dernière narratrice, Shamsa, comprend que le langage qui entoure l'argent et les dons peut parfois signifier autre chose que le désir d'acheter, de posséder ou de mettre l'autre dans sa dette. C'est ce que soutient Jacques Godbout dans sa conférence «Le langage du don » qui se propose de dépasser la théorie de Mauss et d'envisager l'humain comme un être de relation d'abord, et comme être engrené dans la productivité économique par la suite. Épousant la vision de Godbout qui équivaut le don au langage propre de l'amour, Shamsa accepte que «le 
langage du don repose tout simplement sur le besoin d'aimer et d'être aimé qui est aussi fort, et même probablement plus fort et plus fondamental que le besoin d'acquérir » (Godbout 26). Par son dénouement heureux, le premier de son genre, précédé seulement par celui plus optimiste de Je dois tout à ton oubli dans toute la bibliographie de Mokeddem, La désirante, roman paru trente-quatre ans après l'arrivée de l'auteur en France, réconcilie la narratrice avec sa double appartenance à la France et à l'Algérie : « [...] tes mots d'amour rallument les bleus de la mer et ma joie. Le regard encore aimanté par les limbes du Sud, je vois, sans surprise, s'y profiler l'ombre immémoriale d'un vent de sable »(238). On est bien loin des tensions identitaires irrésolues qui déchirent le personnage de Sultana ${ }^{11}$ à la fin de L'interdite: "Maintenant en France, je ne suis ni algérienne, ni même maghrébine. Je suis une Arabe. Autant dire, rien » (191).

\section{Ouvrages consultés}

Aas-Rouxparis, Nicole. «Interdiction et liberté dans L'interdite de Malika Mokeddem ». Malika Mokeddem : Envers et contre tout. Dir. Yolande Aline Helm. Paris : Harmattan, 2000. 157-173.

Brunet, Julie. «Histoires de grands-mères : exil, filiation et narration dans l'écriture des femmes migrantes du Québec ». Cahiers de l'IREF 13. Montréal : UQAM, 2004.

Evans, Jane E. Tactical Silence in the Novels of Malika Mokeddem. Amsterdam : Rodopi, 2010.

Godbout, Jacques. Le langage du don. Montréal : Fides, 1996.

Helm, Yolande Aline. «Entretien avec Malika Mokeddem ». Malika Mokeddem : Envers et contre tout, Dir. Yolande Aline Helm. Paris : Harmattan, 2000. 39-51.

Lazreg, Marnia. The Eloquence of Silence: Algerian Women in Question. New York: Routledge, 1994.

Martin, Florence. «On ne naît pas francophone, nomade et méditerranéenne, on le devient : N’Zid de Malika Mokeddem ». IJFS 5.3 (2003): 173-181.

Mauss, Marcel. « Essai sur le don. Forme et raison de l'échange dans les sociétés archaïques ». Sociologie et anthropologie, 4e éd. Paris : PUF, 1968.

Mehta, Brinda, J. «Geographies of Space : Spatial Impositions, Circularity, and Memory in Malika Mokeddem's Les hommes qui marchent and Le siècle des sauterelles». Meridians : Feminism, Race, Transnationalism 4:1 (2003) : 1-38. 
Mokeddem, Malika. Les hommes qui marchent. Paris : Grasset, 1990.

---. L'interdite. Paris : Grasset, 1993.

---. La nuit de la lézarde. Paris : Grasset, 1998.

---. N'Zid. Paris : Seuil, 2001.

---. La transe des insoumis. Paris : Grasset, 2003.

---. Mes hommes. Paris : Grasset, 2005.

---. Je dois tout à ton oubli. Paris : Grasset, 2008.

---. La désirante. Paris : Grasset \& Fasquelle, 2011.

Sibony, Daniel. Entre-deux. L'origine en partage. Paris : Seuil, 1991.

\section{NOTES}

${ }^{1}$ Selon Brinda J. Mehta, il serait impossible de séparer l'écriture de Mokeddem de la poétique saharienne qui fait du désert un véritable palimpseste de la mémoire ancestrale (5).

${ }^{2}$ Le personnage principal du roman L'interdite, Sultana, s'exile en France pour fuir des interdictions qui dictent la vie des femmes en Algérie. Elle abandonne tout pour être libre, même son grand amour, Yacine, qui deviendra le médecin de leur village natal.

${ }^{3}$ Dans un entretien avec Yolande Helm, Mokeddem parle ouvertement de sa répugnance face à la passivité avec laquelle sa mère a accepté son sort et à l'obstination avec laquelle elle a essayé de l'imposer à sa fille : " J'ai toujours été très violente vis-à-vis de ma mère car j'avais très peur ; elle représentait pour moi tout ce que je n'avais pas envie d'être et j'avais tellement peur de ses menaces, tellement peur de ses injonctions et de ses ordres que je suppose que cette violence aurait pu me détruire, moi d'abord» (41-42).

${ }^{4}$ Cette relation tripartite entre la mère, la fille et la grand-mère apparait dans d'autres textes de l'écriture migrante, comme chez Marie-Célie Agnant, par exemple. Dans la plupart des cas, le rapport est problématique pour la fille, puisque la mère se fait porteuse des enseignements patriarcaux, ce qui lui donne une fausse apparence d'autorité dans la famille et lui fait oublier son propre sort de femme soumise ; en revanche, la grand-mère vient à l'aide de sa petite-fille et la communication entre les deux s'effectue aisément.

${ }^{5}$ C'est nous qui soulignons l'ambigüité grammaticale dans le texte. Il s'agit en fait de la mère de Selma aussi.

${ }^{6} \mathrm{La}$ citation mise en exergue dans le paratexte du roman Je dois tout à ton oubli est un extrait de Médée d'Euripide : « Je préfère lutter trois fois sous le bouclier, plutôt que d'accoucher une seule ».

${ }^{7}$ C'est pourquoi la famille ne permettra à aucune des héroïnes de Mokeddem de leur rendre visite accompagnées par leur conjoint français.

${ }^{8}$ Dans un article intitulé «L'espace-temps de l'écriture migrante au féminin et au masculin » (S. Pruteanu, PluriCulture et écrits migratoires, Monographs in Humanities, Sudbury, 2014), nous développons ce rapport de filiation mal-vécue ou interrompue (par la mort de la mère) et ses influences sur la psyché des femmes migrantes chez Mokeddem et A. Farhoud.

${ }^{9}$ Shamsa est le féminin du nom Shamse qui veut dire « Soleil » en arabe.

${ }^{10}$ Le personnage de Shamsa est du moins contradictoire par rapport à ce sujet. Alors qu'elle refuse d'accepter de l'aide financière, elle fait un don important à la mère d'un de leurs amis, Nabil, à l'insu de celui-ci. En plus, elle pense le récompenser pour son aide dans la recherche de Léo en lui donnant de l'argent pour s'acheter un bateau.

${ }^{11}$ On est également loin de la résignation à la solitude et à la non-appartenance de Nora Carson, personnage de N'Zid, roman qui débute l'écriture des années 2000 chez Mokeddem. Nora essaie de ne pas se laisser piéger par les discours ethnocentriques et elle refuse les ghettos identitaires en affirmant : «Je suis une nomade sans tribu » (176). Fille d'un père d'origine irlandaise et d'une mère algérienne, Nora symbolise l'entre-deux identitaire tout comme la Méditerranée symbolise le nomadisme, le voyage et l'ouverture. 